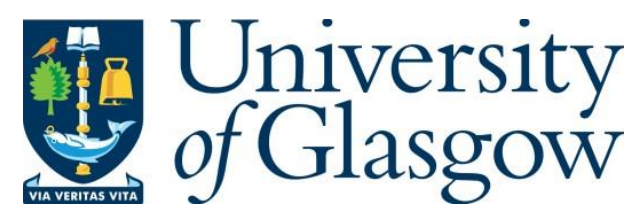

Hoste, J.-J. O., Casseau, V., Fossati, M., Taylor, I. J. and Gollan, R. (2017) Numerical Modeling and Simulation of Supersonic Flows in Propulsion Systems by Open-Source Solvers. In: 21st AIAA International Space Planes and Hypersonics Technologies Conference, Xiamen, China, 06-09 Mar 2017, ISBN 9781624104633.

There may be differences between this version and the published version. You are advised to consult the publisher's version if you wish to cite from it.

http://eprints.gla.ac.uk/140369/

Deposited on: 9 June 2017

Enlighten - Research publications by members of the University of Glasgow http://eprints.gla.ac.uk 


\title{
Numerical Modeling and Simulation of Supersonic Flows in Propulsion Systems by Open-Source Solvers
}

\author{
Jimmy-John O.E. Hoste, ${ }^{*}$ Vincent Casseau, 1 Marco Fossati ${ }^{\dagger}$ \\ Centre for Future Air-Space Transportation Technology, \\ Department of Mechanical and Aerospace Engineering, \\ University of Strathclyde, Glasgow, G1 1XJ, United Kingdom \\ and \\ Ian J. Taylor $\ddagger$ \\ Division of Aerospace Sciences, School of Engineering, \\ University of Glasgow, Glasgow, G12 8QQ, United Kingdom \\ and \\ Rowan J. Gollan 2 \\ Centre for Hypersonics, School of Mechanical \& Mining Engineering, \\ The University of Queensland, Brisbane, QLD 4072, Australia
}

\begin{abstract}
Two open-source solvers, Eilmer and hyFoam, are here considered for their performance in simulating high-speed flows in different flow conditions and geometric configurations typical of propulsive systems at supersonic speeds. The goal is to identify the open-source platform providing the best compromise between accuracy, flexibility and computational cost to eventually simulate the flow fields inside ramjet and scramjet engines. The differences in terms of discretization and solution methods of the selected solvers are discussed in terms of their impact on solution accuracy and computational efficiency and in view of the aerothermodynamic analysis and design of future transatmospheric propulsive systems. In this work steady state problems are considered. Numerical results of two scramjet type engines demonstrated a similar predictive capability of both codes in non-reacting conditions. These results highlight their potential to be considered for further characterization of overall engine performance.
\end{abstract}

\footnotetext{
* PhD Candidate, AIAA Student Member.

${ }^{\dagger}$ Lecturer, AIAA Member .

${ }^{\ddagger}$ Senior Lecturer.
} 


\section{Nomenclature}

$\rho \quad$ density $\left(\mathrm{kg} / \mathrm{m}^{3}\right)$

$u_{i} \quad$ velocity components $(\mathrm{m} / \mathrm{s})$

$\delta_{i j} \quad$ kronecker delta: $0(i \neq j), 1(i=j)$

$p \quad$ static pressure $(\mathrm{Pa})$

$t_{j i} \quad$ molecular stress tensor

$\tau_{j i} \quad$ Reynolds stress tensor

$E \quad$ total energy $(\mathrm{J} / \mathrm{kg})$

$H$ total enthalpy $(\mathrm{J} / \mathrm{kg})$

$I \quad$ turbulence intensity (\%)

$q_{j} \quad$ heat flux components $\left(W / m^{2}\right)$

$Y_{s} \quad$ mass fraction of species $s$

$T \quad$ static temperature $(K)$

$X_{s} \quad$ molar fraction of species $s$

$\mu_{s} \quad$ species dynamic viscosity (Pa.s)

$\kappa_{s} \quad$ species thermal conductivity $\left(W /\left(m^{2} K\right)\right)$

$k \quad$ turbulent kinetic energy $(\mathrm{J} / \mathrm{kg})$

$\omega \quad$ dissipation rate of turbulent kinetic energy $(1 / s)$

$\mu_{t} / \mu$ ratio of turbulent to laminar viscosity

\section{Introduction}

The future of access-to-space systems is faced with many technical and economic challenges. Re-usability has become a main paradigm in the aerospace and space industry as exemplified by the recent Falcon 9 program of SpaceX and the constraints imposed by such requirements further increase the complexity of the design process. Over the years different design solutions have been proposed to realize reliable and cost-effective systems. Canonical solutions for access-to-space systems based on two-, or multi-stage-to-orbit designs improved efficiency while allowing for some degree of re-usability $[1,2]$. The technology of combined cycle propulsion (CCP) systems has allowed switching between different propulsion technologies in a way to ensure the most efficient flight path as exemplified by Marquardt's conceptual design of the Supercharged Ejector RamJet (SERJ) engine[3]. A recent study conducted by NASA showed how rocket-based combined cycle (RBCC) and turbine-based combined cycle (TBCC) engines are technologies that could enable highly-efficient access to space and future airliner propulsion systems preserving full re-usability [4].

The concept of combining together different propulsive systems to optimize the performance according to the operational regime is key to the development of future vehicles. Along this perspective, engineering opti- 
mized systems for the atmospheric high-Mach segment of the flight path is crucial. Ramjet and supersonic combustion ramjet (scramjet) technologies are constantly being studied to target specifically the supersonic and hypersonic regimes thanks to their favourable specific impulse at high Mach numbers. Designing and testing such advanced systems demands the ability to fully control the complex flow physics of these systems. Computational Fluid Dynamics (CFD) and experimental testing have seen great advances through the years in terms of reliability and accuracy. However, in the case of supersonic and hypersonic regimes, realistic high-enthalpy conditions can only be maintained and thus measured in experimental facilities for a very short time, in the order of milliseconds [5,6], and the numerical approach still remains as the primary analysis tool to cover the operating regimes of scramjets in a consistent manner. CFD methods targeting the solution of the flow field inside supersonic engines are faced with the challenges to address internal flows dominated by complex shock wave patterns, shock-wave boundary layer interaction (SWBLI), separation and most importantly supersonic combustion which, in many cases, is characterized by finite rate chemistry. Inaccurate predictions of shock positions and separation locations can lead to unreliable results when reactions are activated putting at risk the success of the entire design process.

A variety of $\mathrm{CFD}$ solvers are present in the literature for the simulation of high-speed air-breathing engines. These include REACTMB[7, 8], WINDUS [9, 10], TAU-code [11], VULCAN [12, 13] and the recent SU2[14, 15]. OpenFOAM [16] is a well-established open platform in the scientific community. Supersonic combustion studies with OpenFOAM-based solvers include the work of Chapuis [17], Fureby [18], Dröske [19] and Makowka [20]. Unfortunately the latter OpenFOAM implementations are not yet fully open-source. The OpenFOAM-based hyFoam ${ }^{1}$ and its variant with a two-temperature model hy2Foam [21, 22], are solvers developed at the University of Strathclyde with the purpose of studying external aerodynamics in re-entry flows around vehicles characterized by high-temperatures effects.

An interesting recent open-source software targeting specifically compressible and potentially chemically reacting flow is Eilmer ${ }^{2}$. It is a solver developed at the University of Queensland. Gollan and Jacobs [23] discussed the main features of Eilmer and validated the solver with several test cases. Chan et al. [24] presented the validation of the implemented $k-\omega$ model in Eilmer with test cases representative for parts of the flow fields inside scramjet engines. Other studies with Eilmer for non-reacting conditions include supersonic cavity flows [25, 26], hypersonic SWBLI [27] and scramjet com-

\footnotetext{
${ }^{1}$ https://github.com/vincentcasseau/hyStrath

${ }^{2}$ http://cfcfd.mechmining.uq.edu.au/eilmer3.html
} 
bustors [28]. In reacting conditions the solver has been used for combustion in narrow channels by Kang et al. [29].

This work represents a first step towards the formulation and implementation of an open-source simulation platform for supersonic and hypersonic propulsion systems where high-fidelity CFD approaches will be coupled with a mission profile analyzer/optimizer to accurately explore the behavior of the propulsion system over a wide range of operating and environmental conditions. Along this perspective, the choice of the most suitable open-source CFD solver that balances accuracy and computational cost is an important factor. The present work presents a comparative assessment of hyFoam and Eilmer for internal supersonic flows to help identify those characteristics of the flow solvers needed to efficiently realize further developments in the open-source simulation framework. As a preliminary step towards this objective, this work will be focusing only on steady state problems.

The material is presented as follows. Section 2 describes the governing equations for fluid flows followed by the main differences between the two solvers. Eventually a series of the test cases will be discussed in Section 3 including proper validation with available reference data. This section will also include results and finally conclusions and future work are detailed in Section 4.

\section{Physical Modelling and Numerical Approaches}

The aim of this work is to assess the predictive capability of Eilmer and hyFoam for scramjet type geometries with a main focus on the accuracy. Results will be influenced by the choice of the discretization schemes and by the models adopted for the different terms appearing in the system of conservation equations.

\subsection{Governing Equations}

The flow established in scramjets is addressed by means of the Navier-Stokes augmented with the conservation equations for each chemical species that constitutes the air-fuel mixture

$$
\begin{gathered}
\frac{\partial \bar{\rho}}{\partial t}+\frac{\partial}{\partial x_{i}}\left(\bar{\rho} \tilde{u}_{i}\right)=0 \\
\frac{\partial}{\partial t}\left(\bar{\rho} \tilde{u}_{i}\right)+\frac{\partial}{\partial x_{j}}\left(\bar{\rho} \tilde{u}_{j} \tilde{u}_{i}+\delta_{i j} \bar{p}\right)=\frac{\partial}{\partial x_{j}}\left(\bar{t}_{j i}+\bar{\rho} \tau_{j i}\right) \\
\frac{\partial}{\partial t}(\bar{\rho} \tilde{E})+\frac{\partial}{\partial x_{j}}\left(\bar{\rho} \tilde{u}_{j} \tilde{H}\right)=\frac{\partial}{\partial x_{j}}\left(\bar{\tau}_{i j} \tilde{u}_{i}+\overline{\tau_{i j} u_{i}^{\prime \prime}}-\bar{q}_{j}-\bar{\rho} \widetilde{H^{\prime \prime}} u_{j}^{\prime \prime}\right)
\end{gathered}
$$




$$
\frac{\partial\left(\bar{\rho} \tilde{Y}_{s}\right)}{\partial t}+\frac{\partial\left(\bar{\rho} \tilde{Y}_{s} \tilde{u}_{j}\right)}{\partial x_{j}}=-\frac{\partial}{\partial x_{j}}\left(\bar{\rho} \widetilde{Y}_{s}^{\prime \prime} u_{j}^{\prime \prime}\right)
$$

where $\bar{\rho}, \bar{\rho} \tilde{u}_{j}, \bar{\rho} \tilde{E}, \bar{\rho} \tilde{Y}_{s}$ are respectively density, momentum, total energy per unit volume and partial densities of the species $s(\mathrm{~s}=1, \ldots, \mathrm{N})$. The symbols $\bar{x}$ and $\tilde{x}$ represent respectively the time and Favre average. Equations 1 to 4 are written in such a way that those terms which require modeling are indicated at the right-hand side. The system of conservation equations for a turbulent chemically reacting flow needs extensive modeling. A comprehensive overview of the modeling practice for supersonic internal flows can be found in the work of Baurle and the interested reader is referred to the literature [30]. In the following paragraphs the main differences in such modeling between the selected solver will be addressed.

\subsection{Eilmer and hyFoam: physical and numerical modeling}

Thermochemistry A thermally perfect gas is usually assumed in ramjet and scramjet flows where the heat capacities are temperature dependent. In hyFoam the species heat capacities are obtained by adding the contributions of the different energy modes [21]. The same is applied for the total energy. Eilmer [31] adopts temperature dependent species heat capacities and energies that are evaluated with the polynomial curve fits of McBride and Gordon [32]. The polynomials rely on coefficients that are species dependent and for this purpose the CEA2[32] library from NASA is used. Given the relatively low temperatures in the targeted applications, the different approach is not expected to greatly impact the flow predictions.

Transport properties The viscosity $\mu_{s}$ and thermal conductivity $\kappa_{s}$ of each species is temperature dependent. In both hyFoam and Eilmer, CEA2 curve fits are used [32]. For the mixture values the former solver uses Wilke's mixing rule [33] while the latter adopts a variant of this same rule [31].

Turbulence model In scramjet flows SWBLI are important features and the turbulence model will play a major role in dealing with it. The linear eddy viscosity two-equation $k-\omega$ turbulence model is the base model for both solvers even if a different variant of it is implemented, and thus available, in each one of them. Eilmer uses Wilcox's $2006 k-\omega$ model $[24,34]$ while hyFoam adopts Menter's Shear Stress Transport (SST) variant $[35,36,37]$. The two-equation models approach is commonly adopted for hypersonic propulsion CFD [38]. This trend follows from the larger range of applicability and suitability for complex geometries in comparison to e.g. one equation models without drastically increasing the computational cost 
[30]. The development of the SST model was motivated by the sensitivity of the $\mathrm{k}-\omega$ model to freestream turbulence properties. Away from walls the model uses a $\mathrm{k}-\epsilon$ turbulence formulation and switches to k- $\omega$ when approaching physical boundaries. In a SWBLI study reported by Georgiadis et al. [38] the SST model had a tendency to overpredict the flow separation when comparing with experimental PIV data and other turbulence models (no comparison with $2006 \mathrm{k}-\omega$ ). Over the years the freestream sensitivity limitation of the $\mathrm{k}-\omega$ has been addressed and improvements have been made. The improvements in the 2006 version implemented in Eilmer include: reduced freestream sensitivity, improved behavior for compression corners and hypersonic SWBLI and the capability to handle free shear flows [34]. For this latter model the importance of the stress limiter parameter in predicting separation was demonstrated by Wilcox [34] and confirmed by Chan et al. [24] and reasonable agreement with wall pressure data was observed. This version is capable of similar performances as Menter's SST model and the effect of the choice in each solver will be closely observed in the comparison test cases with special attention to separation in SWBLI regions.

The Finite Volume Discretization Both solvers adopt a Finite Volume discretization for the governing equations where the inviscid and viscous fluxes are treated separately. The main difference between Eilmer and hyFoam is observed in the evaluation of the inviscid fluxes. In Eilmer the inviscid fluxes are obtained with a so-called adaptive method which switches between the Equilibrium Flux Method (EFM) and an Advection Upstream Splitting Method combining difference and vector splitting (AUSMDV). The EFM method [39] is used in the vicinity of shock waves and being derived from the kinetic theory is inherently upwind which makes it suitable for highly compressible flows. In the other regions Wada and Liou's [40] AUSMDV method is preferred due to its less dissipative character. The AUSMfamily schemes have received much attention in the CFD community thanks to the wide range of applicability from low to high Mach number and to multiphase flows[41]. In a comparison study by Coratekin et al.[42] the authors observed a better performance of the AUSMDV scheme in hypersonic flow conditions compared to the original AUSM scheme and Roe's flux difference splitting scheme. Eilmer uses explicit time-stepping on structured grids. The hyFoam is an unstructured solver developed in OpenFOAM 2.3 originating from two available solvers: rhoCentralFoam and reactingFoam. This latter is a combustion solver that has multi-species libraries while rhoCentralFoam is a single-species density-based compressible solver which makes use of the central-upwind schemes of Kurganov and Tadmor [43, 44]. The use of these flux schemes for propulsive supersonic flow problems has not yet been thoroughly evaluated. The motivation of Kurganov and Tadmor $[43,44]$ in introducing a central scheme lies in the fact that the method does 
not rely on the specific eigenstructure of the problem. The central-upwind character follows from the calculation of a weighting coefficient that uses one-sided local speeds of propagation. Greenshield et al. [45] implemented the method within OpenFOAM and similar predictions to a Roe solver were obtained for the separation region on a hypersonic flow over a double cone configuration. The implementation combines an explicit predictor step for the convection of the conserved variables followed by an implicit corrector step for the diffusion of primitive variables.

The numerical approach in hyFoam has been validated for laminar supersonic and hypersonic steady state flows but has not yet been used in conjunction with turbulent models. It has the potential to extend its scope to address scramjet-like internal flows in an unsteady fashion and the choice of the OpenFOAM framework could benefit from the contribution of the wide OpenFOAM community. This includes turbulent modelling, Finite Volume discretization approach, limiting functions and parallelization. The hyFoam offers the possibility to use unstructured grids but to the knowledge of the authors the use of Kurganov and Tadmor's scheme has not been validated by other OpenFOAM studies with such grids. Eilmer on the contrary has been widely assessed and validated for turbulent problems representative of scramjet internal aerothermodynamics but has a more restricted developers community compared to OpenFOAM. The developments are however targeted to the specifics of high speed internal and external flows which is not the case in the OpenFOAM framework. With regard to the final objective, Eilmer and hyFoam possess the capabilities to be considered for further studies in scramjets flow physics and performance. Current limitations in Eilmer are the structured and the explicit character of the code. Unstructured grids allow more complex geometries to be simulated and can become a necessity when Rectangular-to-Elliptic-Shape-Transition scramjet [28] types are considered. Explicit solvers are known to increase the computational cost in steady state problems. A new version of the Eilmer code ${ }^{3}[46]$ is currently being developed which will address both limitations. This study will do a thorough investigation of the above introduced numerics and model choices for the flows of interest on Cartesian grids.

\section{Test Cases}

Results refer to two test cases available in the literature for typical propulsive applications: the first is from Lorrain [6] and the second is from Smayda et al. [47]. All simulations have been made considering the same grid for the different solvers, thus allowing a grid-independent comparison.

\footnotetext{
${ }^{3}$ https://bitbucket.org/cfcfd/dgd
} 


\subsubsection{Lorrain's Scramjet}

The scramjet's internal flow is dominated by shock wave reflections interacting with boundary layers. This test case asses the capability of both CFD solvers in predicting a shock train and allows evaluation of the numerical diffusion of the spatial discretization schemes. The selected scramjet geometry is a test case investigated by Lorrain et al. [6, 48] in the University of Queensland's T4 hypersonic piston-driven shock tunnel. It is a specific type of scramjet engine which relies on the concept of radical farming and has been introduced within the the SCRAMSPACE I project [49]. The same geometry has previously been simulated with OpenFOAM $2.1^{4}$ by Mogavero et al. [50] using the Weighted Average State (WAS) method [51]. Since the development of this solver called rhoFoam, OpenFOAM has had major changes in the thermophysical modeling.

Problem formulation Figure 1 shows the geometry and boundary conditions used by Lorrain et al. [6] for study of the finite-rate chemistry mechanisms governing the combustion process with CFD $++[52]$. As it can be seen in the upper representation, the full geometry consists of an inlet (sharp leading edge) with two ramps leading to the constant area combustor which terminates in an exit nozzle. As a numerical demonstration, a premixed configuration (lower representation) was also studied. Lorrain aimed at investigating shock and expansion wave structures as well as boundary layer viscous heating effects. The test case has been simulated in three different conditions $[6]$

a. A full geometry (upper part of Figure 1) with only air as a working fluid.

b. A frozen hydrogen fueled simulation with only the combustor and nozzle (lower part of Figure 1).

c. A reacting hydrogen fueled simulation with only the combustor and nozzle (lower part of Figure 1).

The mesh grading used in the presented simulations ensured a $y^{+}$value smaller than unity. In the fuel-off test case the number of cells in the first intake, second intake, combustor and nozzle were respectively equal to 110 $\times 456,110 \times 300,110 \times 1454$ and $110 \times 718$ resulting in a total of 322,080 cells with a maximum aspect ratio of 340 . For the frozen and combusting simulations the mesh size was reduced to 133,414 cells with a maximum aspect ratio of 501 to enable a quicker analysis. From the final report of Lorrain [53] (Table 7.1 without symmetry assumption) the grid sizes selected in the current study are considered to be adequate. Table 1 summarizes

\footnotetext{
${ }^{4}$ http://openfoam.org/release/2-1-0/
} 


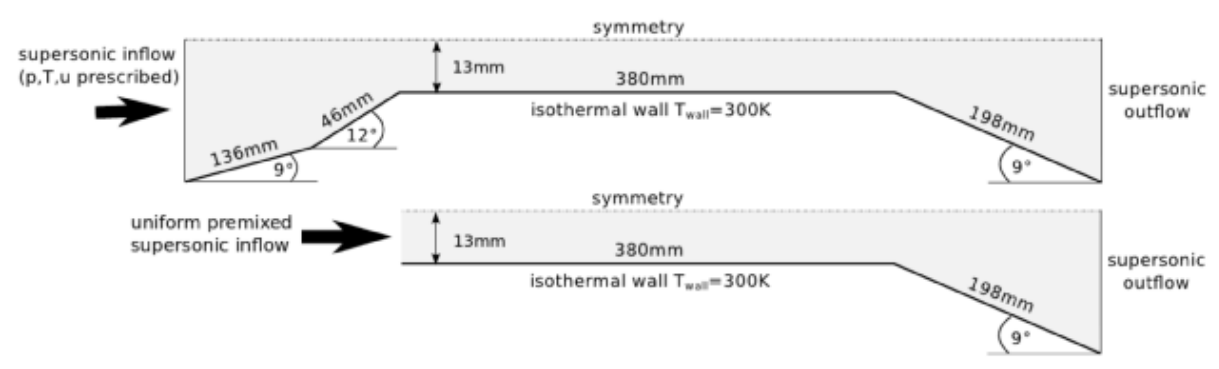

Figure 1: The geometry considered for scramjet CFD simulations [6] with top: for fuel-off case, bottom: for frozen and combusting case

the flow conditions of Lorrain's experimental and numerical study for the fuel-off case with $\mathrm{u}, \mathrm{p}, \mathrm{T}$ and $\mathrm{X}$ being respectively the velocity, static pressure, static temperature and mole fractions. Turbulent inlet quantities were set with an intensity $I$ of $2 \%$ and a ratio of viscosities $\mu_{t} / \mu$ equal to 5 . A thermally perfect gas is assumed with heat capacities depending on the temperature. The symmetry of the geometry was exploited in the simulations and the walls are considered isothermal at a temperature of $300 \mathrm{~K}$. This assumption is acceptable considering the very short experimental test time, on the order of milliseconds, which is not sufficient to cause significant temperature elevations. A turbulent Prandtl number of 0.89 was specified in both solvers for the presented results.

Table 1: Flow conditions at the inlet of the scramjet geometry for the different simulations [6].

\begin{tabular}{l|llllll} 
& $\mathrm{u}(\mathrm{m} / \mathrm{s})$ & $\mathrm{p}(\mathrm{Pa})$ & $\mathrm{T}(\mathrm{K})$ & $X_{O_{2}}(-)$ & $X_{N_{2}}(-)$ & $X_{H_{2}}(-)$ \\
\hline fuel-off & 2830 & 4100 & 370 & 0.21 & 0.79 & 0.0 \\
frozen / reacting & $\mathrm{u}(\mathrm{y})$ & $\mathrm{p}(\mathrm{y})$ & $\mathrm{T}(\mathrm{y})$ & 0.157 & 0.593 & 0.25
\end{tabular}

Results Firstly results have been obtained for test case "a", a set-up with only the presence of air. The pressure contour plot in Figure 2 shows a very similar predictive capability between both solvers.

In order to better distinguish the results from both codes, the wall pressure coefficient is presented in Figure 3 with the experimental data of Lorrain et al. [6]. CFD solution from rhoFoam [50] is displayed as well in order to compare Eilmer and hyFoam with a Riemann solver-based scheme. In hyFoam, the simulations were performed using two different limiter functions for flux interpolation (Gamma1 and van Leer) in order to evaluate appropriate settings for scramjet type flow problems. Overall the two CFD solvers present a very similar profile. Differences in shock positions only start to be 


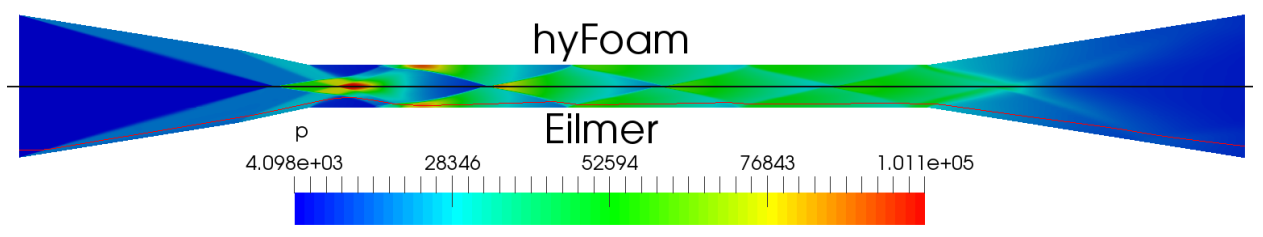

Figure 2: The contour plot of pressure shows a similar shock structure inside the geometry between hyFoam and Eilmer

visible after the entrance of the combustor. Comparing with the experimental data, the simulations seem to underestimate pressure values such as the first peak inside the combustor. Nevertheless, the location is estimated in a fair manner by all solvers. This single peak is the result of a very close reflection of the two intake ramps shocks which then propagate as a single shock. This is more clear in the Schlieren observations provided further below. The combustor profiles do follow the trend of the experimental data but from the second peak onwards a shift in position is observed. The nozzle section predictions seem to miss some fundamental behavior but comparable pressure values are obtained by the different CFD solvers. A closer look at the combustor section in Figure 4 shows a more pronounced discrepancy between Eilmer and the OpenFOAM solvers (rhoFoam and hyFoam) from the second shock impingement location about $16 \mathrm{~cm}$ downstream of the combustor entrance. A slightly better agreement with experimental pressure is obtained using hyFoam and rhoFoam. At the end of the combustor, Eilmer, showing a wider reflection peak (fourth shock reflection inside the combustor), is in closer agreements with experiments. Investigation of the limiters in hyFoam showed a more oscillatory behavior of van Leer compared to Gamma1 for a same CFL setting of 0.1 . Gamma [54] belongs to the category of normalised variable diagram (NVD) schemes while van Leer is a total variation diminishing (TVD) scheme. The setting Gamma1 in hyFoam is the upper limit of this scheme characterized by more numerical diffusion and this is thought to be the explanation of a smoother pressure profile compared to van Leer. On the other hand, results did not differ much except near the end of the combustor with a much shorter and later pressure peak prediction.

At the end of the nozzle (Figure 3) a weak shock reflection is captured by Eilmer and hyFoam (more pronounced for the former solver) which is barely seen in rhoFoam. The reason could be the use of a different mesh in the latter solver. A closer look at the physics in the region near the combustor entrance explains the onset of the difference in positions between Eilmer and hyFoam discussed above. Figure 5 shows the presence of a shock induced boundary layer separation bubble which was also observed by Lorrain [53]. The hyFoam results are obtained with the Gamma1 limiter which gave the 


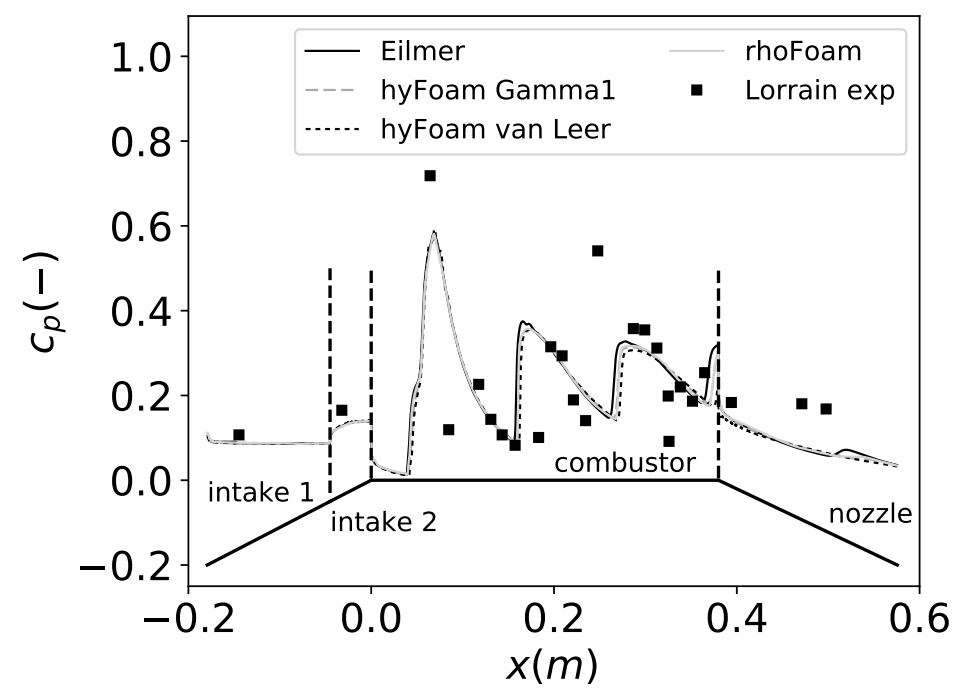

Figure 3: Wall pressure profiles for the fuel-off test case, comparison with experimental values

most satisfactory wall pressure profile and non-oscillatory behavior. The location of the separation region slightly differs $(\approx 3.5 \mathrm{~mm})$ as well as the size. This latter behavior in hyFoam is expected as the $k-\omega$ SST model tends to overpredict the size of SWBLI regions as pointed out by Georgiadis et al. [38]. In terms of turbulent kinetic energy, Eilmer does predict higher values in this region. More detailed experimental data is required in the separation region to evaluate the prediction by Wilcox $2006 \mathrm{k}-\omega$ model but, in accordance with other observations of similar flows [38], a smaller separation region should be captured. A quantitative analysis of the shock structures will help in understanding these results. Figure 6 presents the experimental Schlieren of intake regions and combustor entrance as well as the CFD gradients of density. The different flow features shown experimentally are visible in the numerics with the two shocks of the intake ramps as well as the expansion wave at the combustor entrance corner which is consistent with the previously observed wall pressure profile. The intersection point of the entrance shock with the symmetry plane is predicted slightly earlier in Eilmer compared to hyFoam. Given that the simulations are performed on the same grid this observation suggests the cause of a different shock angle to be related to the numerical schemes treatment of the inviscid fluxes. This original difference explains the shift in position of the separation bubble and consequently the pressure peaks of Figure 4. It must be noted that, when looking at the experimental Schlieren further downstream in the combustor, the reflected shocks do not cross at the center of the geometry. This suggests 
an asymmetry in the geometry which was later confirmed[53]. Consequently the pressure data also captured this asymmetry which is not accounted for in the present simulations, hence explaining partly the mismatch between experimental data and CFD. From the fuel-off analysis, no conclusion can be drawn on the better performance in accuracy of one solver over the other.

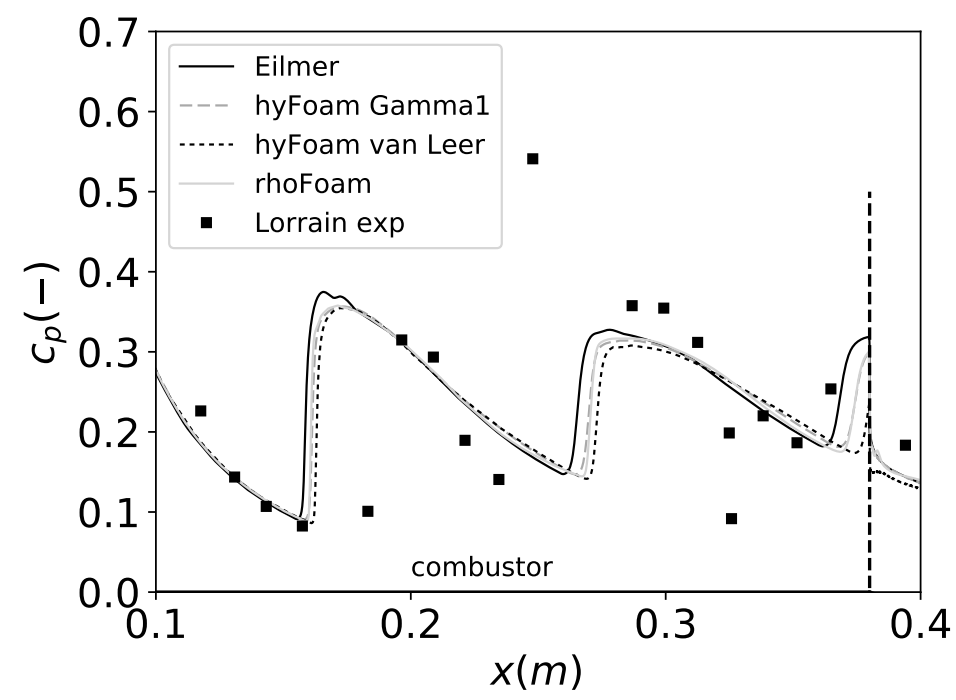

Figure 4: Combustor wall pressure close-up view as predicted by hyFoam and Eilmer

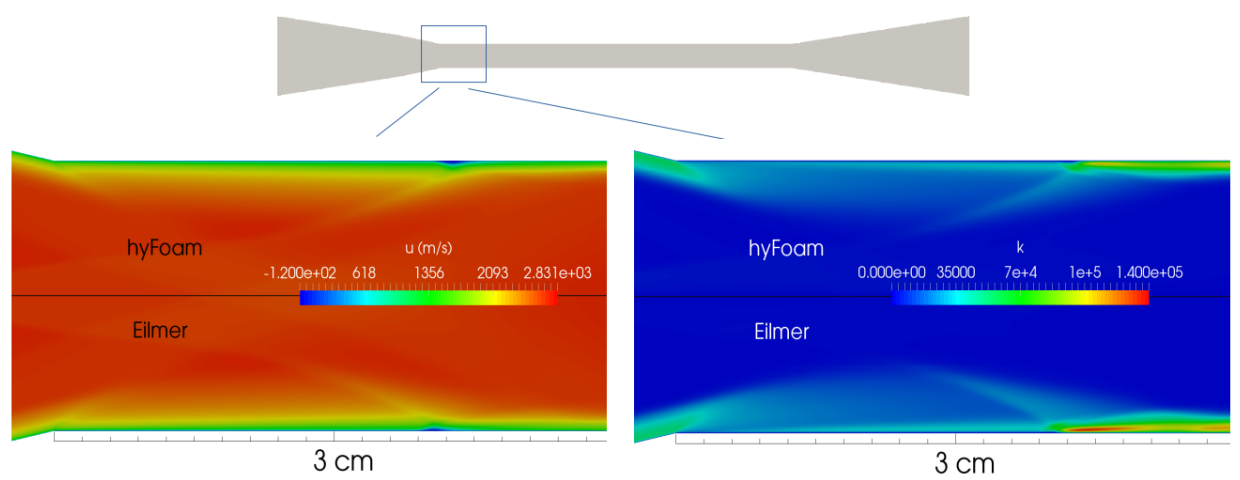

Figure 5: Shock induced boundary layer separation with contour of velocity and turbulent kinetic energy for both solvers 


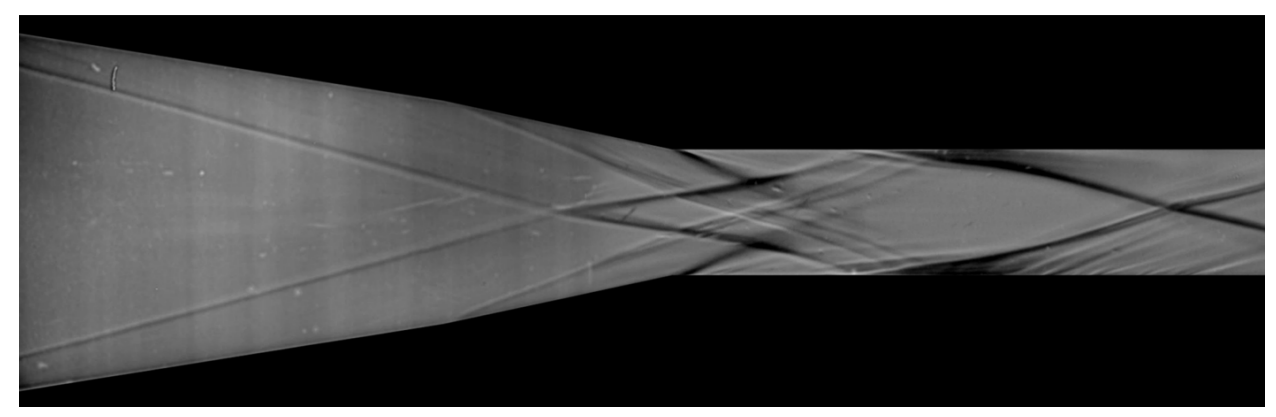

(a) Experiment [6]

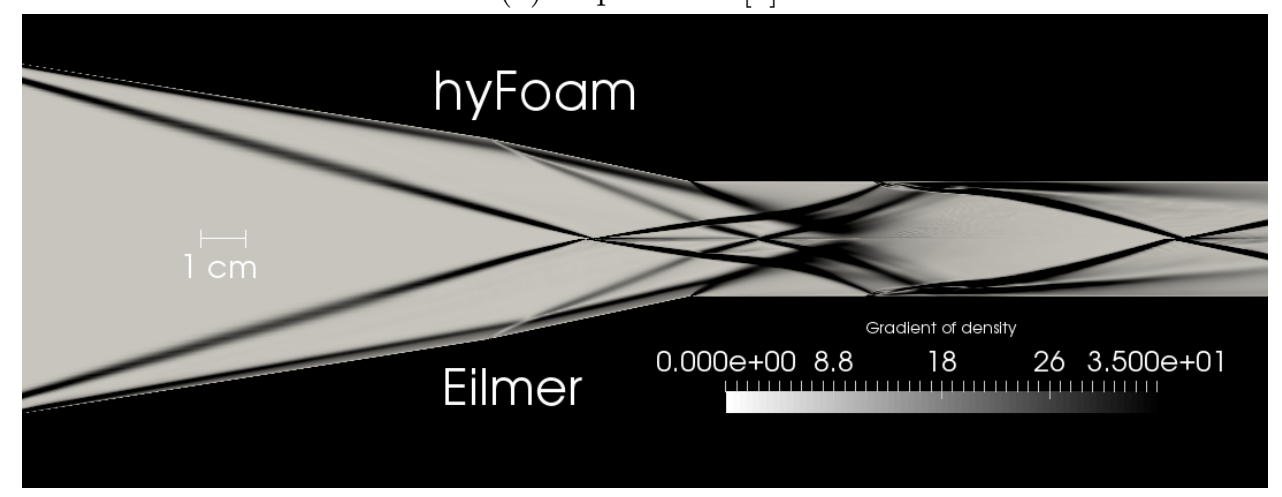

(b) CFD

Figure 6: A comparison of the experimental Schlieren and CFD for the fuel-off conditions. Note that the scales do not perfectly match.

For the simulations in frozen conditions the profiles of the flow variables at the entrance of the combustor are extracted from the fuel-off results and prescribed as inlet boundary conditions. In order to account for the presence of hydrogen the pressure profile was altered as $p_{\text {frozen }}=p_{\text {fuel-off }} /\left(1-X_{H_{2}}\right)$. Figure 7 shows the inlet profiles of temperature and velocity on the left and right side respectively. A symmetry plane is at the top and the wall at the bottom. The velocity is very similar between both solvers but the temperature shows a significant increase in peak value in the boundary layer for hyFoam. A similar behavior is observed for turbulent kinetic energy. The difference seems to be a consequence of the turbulence model. For the frozen condition CFD data of pressure is available from Lorrain et al. [6] and Mogavero et al. [50] along a streamline originating $1.5 \mathrm{~mm}$ from the lower wall at the combustor entrance as shown in Figure 8. The hyFoam data is shown for van leer limiter only as simulations with gamma1 were not completed at the time of writing. It is interesting to see that the results of three different CFD codes (CFD++, rhoFoam and hyFoam) on different grids are very similar. Shock positions in Eilmer slightly differ but no data is available to assess the correctness of the observed profile. As the other three 
solvers use different discretization methods but all rely on the use of the k- $\omega$ SST model, the discrepancy with Eilmer is thought to be a consequence of this turbulence model choice. This conclusion is coherent with the observed temperature profile in Figure 7.

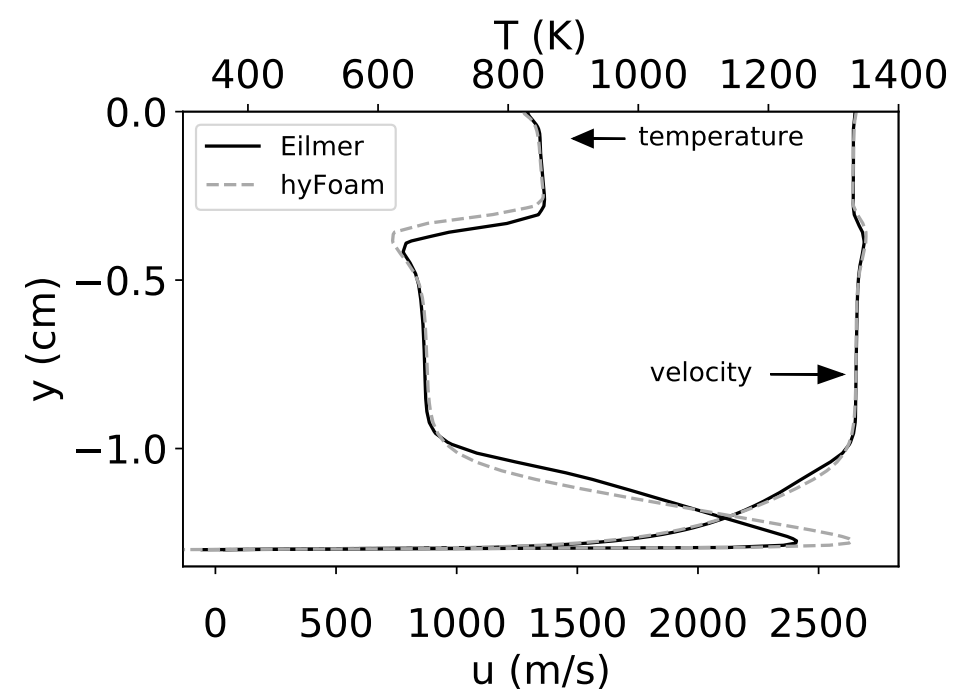

Figure 7: Temperature and velocity profiles at the entrance of the combustor for Eilmer and hyFoam.

In terms of computational cost, hyFoam was observed to have higher global time steps than Eilmer. This behavior is expected given the fully explicit character of the latter solver. In order to quantify the difference the fuel-off test case was run from the converged steady state solution for an extra 10,000 steps and a CFL setting of 0.5 in both solvers on the ARCHIEWeSt (Academic and Research Computer Hosting Industry Enterprise in the West of Scotland) High Performance Computer [55]. The run used 48 Intel Xeon X5650 2.66 GHz cores (Santa Clara, CA, USA) with 48 GB RAM and $4 \mathrm{xQDR}$ Infiniband Interconnect computer-networking communications. The hyFoam reached 10,000 iterations after a wall-clock time of 1396 sec. In Eilmer it took $2700 \mathrm{sec}$ to perform the same number of iterations. The advantage of working with a combination of explicit and implicit schemes in hyFoam is demonstrated. The new version of Eilmer will address this current limitation. It must be noted that conclusions on convergence speed and grid requirements cannot be drawn based on the studies in this work. 


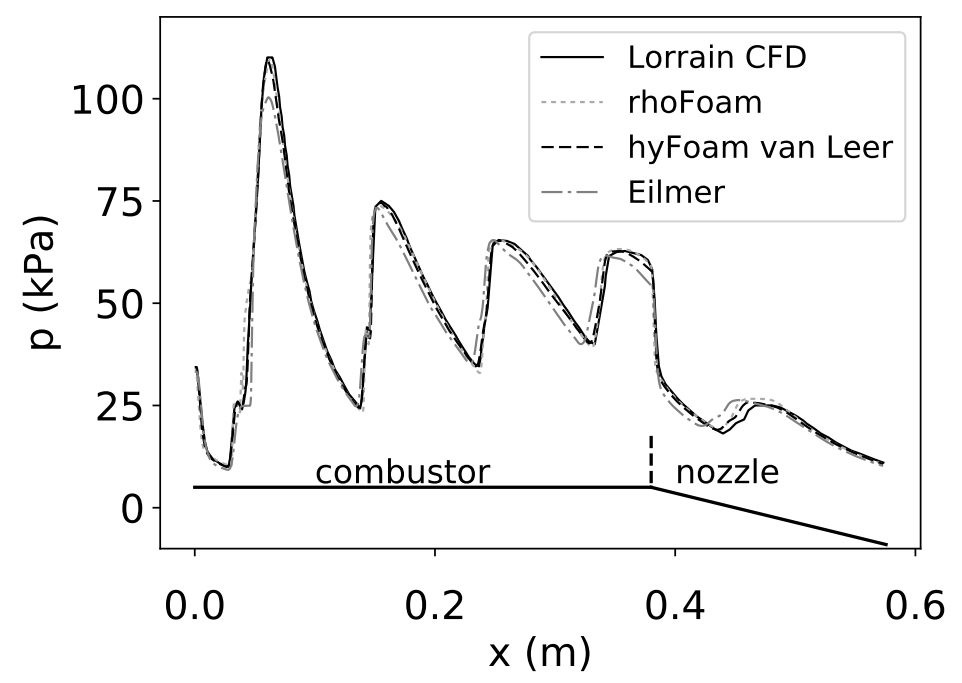

Figure 8: Static pressure of different CFD codes along a streamline starting $1.5 \mathrm{~mm}$ from the combustor's entrance wall

\subsubsection{Hypervelocity Dual-Mode Scramjet}

Another type of scramjet flow has been selected for the comparison of both codes. The scramjet design is a dual-mode scramjet and has been studied experimentally by Smayda et al. [47] in NASA's HyPulse facility. Their interest focused in the higher velocity regimes where pure scram-mode was expected also referred to as hypervelocity (above Mach 7 flight enthalpy conditions). Vogel [56] performed the CFD simulations with REACTMB, North Carolina State University's in-house solver, to compare with the experiments. The following numerical study considers pure scram-mode.

Problem formulation Figure 9 shows a side view of the dual-mode scramjet geometry. The geometry is inspired by the University of Virginia directconnect Dual Mode Scramjet (DSMJ). The inlet section (red) consists of a forebody wedge and inlet cowl redirecting the flow to a constant area rectangular isolator section (blue + purple). Following this shock train section, the flow enters the combustor and is accelerated through the exit nozzle (orange) after fuel injection (green). As the present study focuses on two-dimensional simulations only the inlet (red) and isolator (blue) regions are considered. Near the entrance of the combustor chamber (purple) three-dimensionality becomes important. Vogel [56] simulated the test case at equivalent flight conditions of Mach 7 and 10 with a dynamic pressure of $47.88 \mathrm{kPa}$ (1000 psf). 


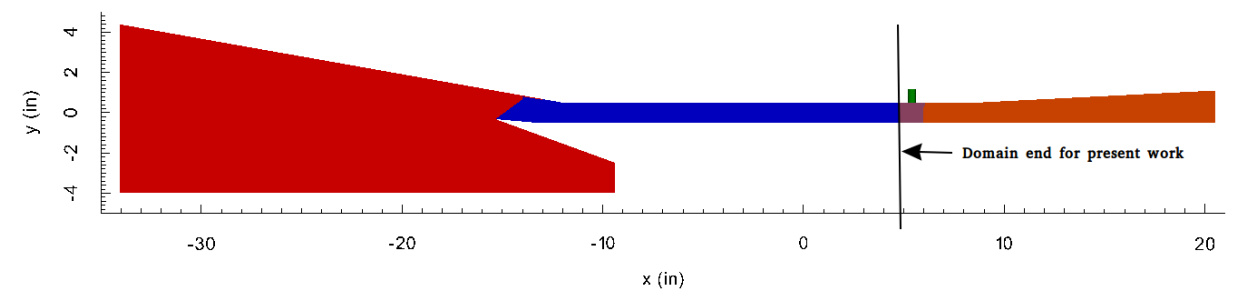

Figure 9: The dual-mode scramjet geometry studied in NASA's HyPulse facility $[47,56]$.

The geometry and mesh configuration used in the present numerical study with Eilmer and hyFoam are given in Figure 10. The mesh contained 594,000 cells with a maximum aspect ratio of 722 . Grading towards the walls ensured $y^{+}$values lower than unity. The Mach 7 equivalent flight test conditions are recreated in this work. A 0.93 degrees angle of attack was considered in the simulations [56] yielding two velocity components as given in Table 2. Freestream turbulence levels were set to $5 \%$ and 10 for $I$ and $\mu_{t} / \mu$, respectively. The walls are considered isothermal at $300 \mathrm{~K}$. Just like in Lorrain's test case, the limited test time did not allow for significant heating of the geometry. Turbulent Prandtl number was set to 0.89 in both solvers.

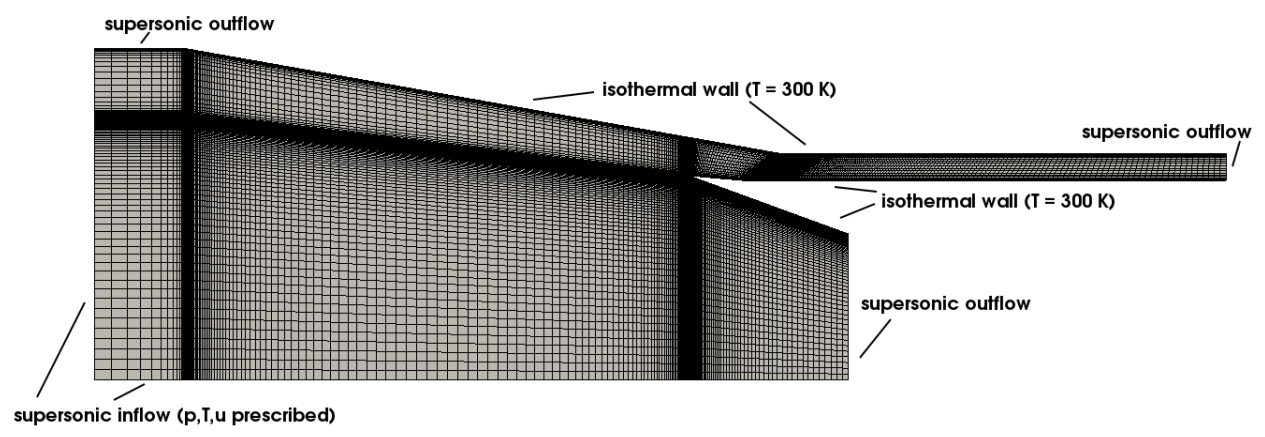

Figure 10: Geometry and boundary conditions for the numerical study of the dual-mode scramjet. Each 4th line is represented in every direction for clarity.

Table 2: Flow conditions at the inlet of the dual-mode scramjet geometry for Mach 7 equivalent flight simulations [56].

\begin{tabular}{l|llllll} 
& $\mathrm{u}(\mathrm{m} / \mathrm{s})$ & $\mathrm{v}(\mathrm{m} / \mathrm{s})$ & $\mathrm{p}(\mathrm{Pa})$ & $\mathrm{T}(\mathrm{K})$ & $Y_{\mathrm{O}_{2}}(-)$ & $Y_{N_{2}}(-)$ \\
\hline Mach 7 & 2122.0 & 34.5 & 1179.0 & 208.9 & 0.24 & 0.76
\end{tabular}


Results Figure 11 presents the gradients of density as predicted by both solvers. More complex shock wave interactions are present at the entrance of the isolator in comparison with Lorrain's test case. On this representation the different flow features of this test case are clearly visible with a forebody shock, an inlet shock redirected by the cowl, an expansion fan, a compression corner and the SWBLI responsible for a separation bubble. Some clear difference can be seen on how the latter is captured by both codes. A thicker separation is present in hyFoam which relies on the k- $\omega$ SST and this flow feature influences the downstream shock train.

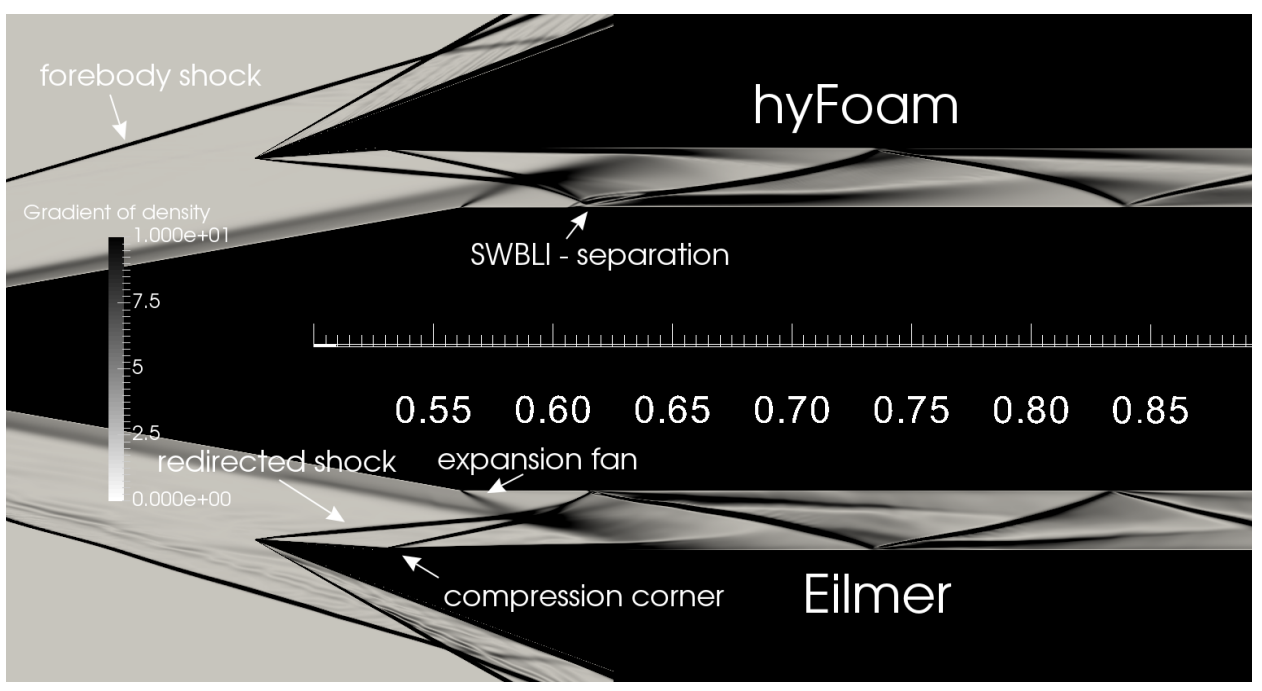

Figure 11: Gradient of density inside the dual-mode scramjet's isolator for hyFoam and Eilmer

Experimental wall pressure data at the injector side is available to assess the accuracy of the CFD simulations. Figure 12 presents the resulting profiles of Eilmer and hyFoam. Following the observations in Lorrain's study, simulations with Gamma1 have been performed for the latter solver. For comparison purposes wall pressure of Vogel [56] with the k- $\omega$ SST model is displayed. Vogel reported as well simulation results with Menter's baseline (BSL) $\mathrm{k}-\omega$ model and observed slightly higher peak values in the isolator section and attributed this to a discrepancy in the SWBLI of both models. Note that Vogel's simulations are three-dimensional and a spillage region is used near the inlet which is not accounted for in this work. The forebody wall pressures are very similar between all solvers. Shock positions start to differ after the start of the expansion fan as seen in Figure 13 where a closer agreement with experimental value $(x \approx 0.61 \mathrm{~m})$ is achieved by Vogel in comparison to Eilmer and hyFoam. In terms of physics this implies that the reflection point of the shock redirected from the inlet cowl interacting with the expansion fan induced at the entrance of the isolator is different. A 
wider expansion region is predicted by both solvers studied in this work and the reflection occurs slightly earlier in hyFoam. The origin of the discrepancy in wall pressure between Eilmer and hyFoam is justified by a different numerical prediction of a shock induced boundary layer separation bubble for an x-position close to $61 \mathrm{~cm}$ as was shown in Figure 11. The isolator entrance shocks of both solvers are very similar as seen in Figure 14 which is a profile of Mach number across the isolator taken at $\mathrm{x}=55 \mathrm{~cm}$. This demonstrates that the SWBLI at $\mathrm{x}=0.61 \mathrm{~m}$ is the main cause for the shift in the downstream shock train. Temperature profiles in Figure 15 along the same vertical line show again higher peak values in the boundary layer regions and relates to the levels of turbulent kinetic energy.

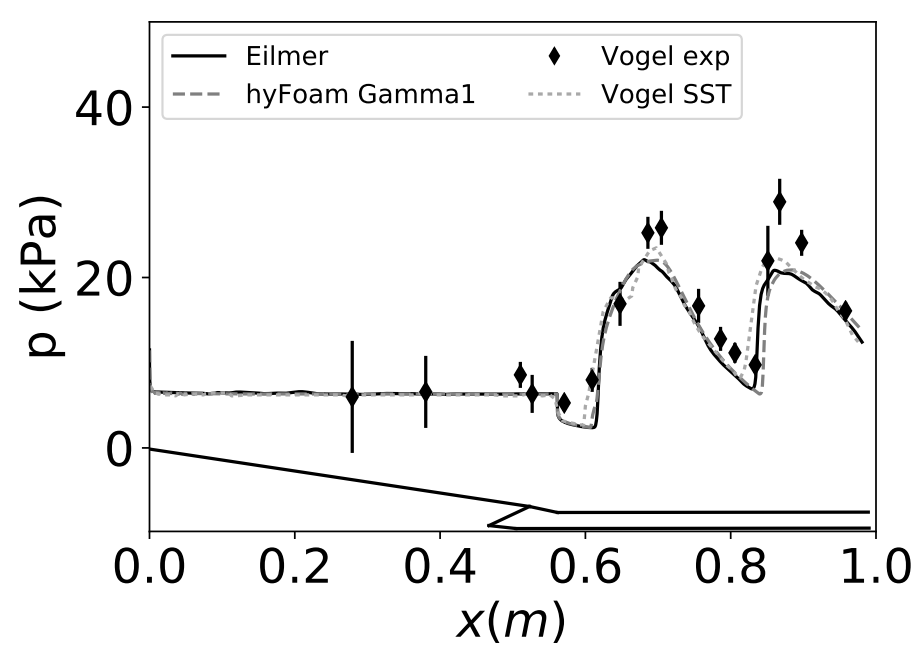

Figure 12: Complete geometry wall pressure comparison of CFD with experiment at injector side

After the SWBLI, the wall pressure (Figure 13) profiles of Eilmer and hyFoam have a similar trend until the next reflection at $x \approx 0.84 \mathrm{~m}$. At that position hyFoam predicts a reflection further downstream compared to Vogel SST and Eilmer. The latter solver agrees better with the experimental values. The gradient in pressure after the reflection is very similar in hyFoam and Eilmer. Consequently the shift in pressure profile originated in this reflected region persists until the end of the geometry. In comparison to Vogel SST both solvers underpredict the peak values. Moreover, between the first and second reflection the reference CFD seems to capture a local minimum which is not captured on the mesh used by the present authors. Following these wall pressure observation with Eilmer and hyFoam, the Wilcox k- $\omega$ 


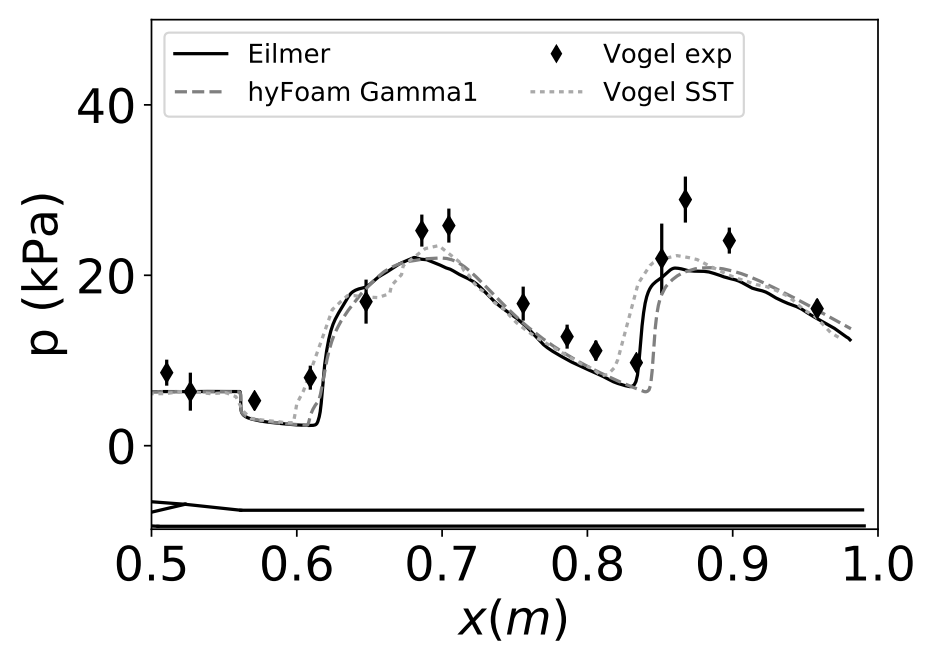

Figure 13: Isolator wall pressure comparison of CFD with experiment at injector side
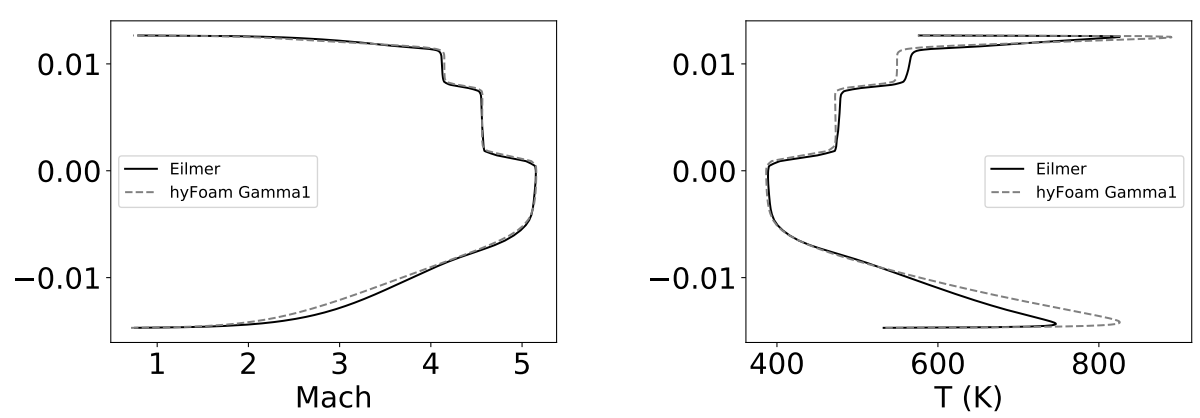

Figure 14: Mach number profiles of Figure 15: Temperature profiles of Eilmer and hyFoam at $\mathrm{x}=55 \mathrm{~cm}$ for Eilmer and hyFoam at $\mathrm{x}=55 \mathrm{~cm}$ for the dual-mode scramjet test case. the dual-mode scramjet test case.

model seems to be more appropriate than the Menter SST version.

Just like in the Lorrain test case, larger global time steps were observed for hyFoam compared to Eilmer which should result in reaching a converged steady-state solution more rapidly. An objective comparison between both solvers to quantify this for the dual-mode scramjet test case has not been made. 
Both the Lorrain and the above test case have shown the importance of the turbulence model on the resulting shock structure inside an isolator. This observation became clear from the typical shock induced boundary layer separation bubble found near the entrance of scramjet type geometries. The early occurrence of such a physical feature influences the downstream shock train. Wilcox $2006 \mathrm{k}-\omega$ model, which predicts a smaller separation bubble compared to the k- $\omega$ SST of Menter, does seem to be a more appropriate choice. This statement is supported by the evolution of the wall pressure data in the dual-mode scramjet geometry. It must be demonstrated how much effect the turbulence model choice has has when a mixture enters a combustion chamber where the flow remains supersonic and mixes with fuel. The spatial discretization schemes of both solvers are observed to play a secondary role as compared with the choice of the turbulence model on the final solution with only small differences observed between Eilmer and hyFoam. Consequently this study confirmed the capability of hyFoam in addressing steady state supersonic internal flows. Improvements could be obtained for the test cases considered in this work by implementing the Wilcox $2006 \mathrm{k}-\omega$ turbulence model. In terms of computational cost, hyFoam was observed to be less demanding than Eilmer. However, given the availability of Wilcox $2006 \mathrm{k}-\omega$ turbulence model, the previously validated combusting capability [29], and the developments on the way to improve the performance (unstructured and implicit) of the code for high speed internal and external flows, Eilmer will be selected by the authors for future studies.

\section{Conclusion}

Two open-source CFD codes Eilmer and hyFoam are considered to simulate the physics of supersonic flows for propulsion applications in a steady state. The assessment is performed with two scramjet type test cases on the same computational grids. Regarding the computational cost hyFoam did perform better than Eilmer which was expected given the fully explicit character of the latter code. In terms of accuracy both solvers have a similar prediction capability when no chemical reactions are considered. The spatial discretization treatment resulted in only slight differences in inlet shock predictions. An important discrepancy is observed in the shock induced boundary layer separation bubble, typical of scramjet flows. The reason for this discrepancy is thought to be related the use of Wilcox 2006 (Eilmer) and Menter's SST (hyFoam) k- $\omega$ turbulence model. Observations on the impact of the separation region on the downstream shock train with experimental data suggest the use of the former turbulence model to be more adequate. It must be noted that this study needs to be pursued further to include also the effect of mesh resolution in order to eventually compare mesh independent results for both solvers. Nevertheless, both solvers demonstrate a capability 
to be used as design tools and in a next step the effect of combustion on the overall engine performance will be evaluated.

\section{Acknowledgments}

The authors would like to express their gratitude to Dr. Jack Edwards from North Carolina State University for the dual-mode scramjet geometry details. A special thanks to Tom J. Scanlon from the University of Strathclyde and Peter Jacobs from the University of Queensland for their advice during the process of realizing this work. Results were obtained using the EPSRC funded ARCHIE-WeSt High Performance Computer (www.archiewest.ac.uk). EPSRC grant no. EP/K000586/1.

\section{References}

[1] Bruno, C. and Czysz, P. A., Future spacecraft propulsion systems: enabling technologies for space exploration, Springer Science \& Business Media, 2009.

[2] Jazra, T. and Smart, M. K., "Design Methodology for the Airbreathing Second Stage of a Rocket-Scramjet-Rocket Launch Vehicle," 17th AIAA International Space Planes and Hypersonic Systems and Technologies Conference, San Francisco, California, 2011.

[3] Escher, W. and Flornes, B., "A Study of Composite Propulsion Systems for Advanced Launch Vehicle Applications," Tech. rep., Vol 7, The Marquardt Corporation, Van Nuysm CA, 1966.

[4] Mankins, J., "Highly reusable space transportation: Advanced concepts and the opening of the space frontier," Acta Astronautica, Vol. 51, No. 10, 2002, pp. 727-742.

[5] O'Byrne, S., Doolan, M., Olsen, S., and Houwing, A., "Measurement and imaging of supersonic combustion in a model scramjet engine," Shock Waves, Vol. 9, No. 4, 1999, pp. 221-226.

[6] Lorrain, P., Capra, B., Brieschecnk, S., and Boyce, R., "A Detailed Investigation of Nominally 2-D Radical Farming Scramjet Combustion," 18th AIAA/3AF International Space Planes and Hypersonic Systems and Technologies Conference, 2012, p. 5812.

[7] Edwards, J. R., Boles, J. A., and Baurle, R. A., "Large-eddy/Reynoldsaveraged Navier-Stokes simulation of a supersonic reacting wall jet," Combustion and Flame, Vol. 159, No. 3, 2012, pp. 1127-1138. 
[8] Edwards, J. R. and Fulton, J. A., "Development of a RANS and LES/RANS Flow Solver for High-Speed Engine Flowpath Simulations," 20th AIAA International Space Planes and Hypersonic Systems and Technologies Conference, 2015, p. 3570.

[9] Engblom, W., Frate, F., and C.C., N., "Progress in validation of WINDUS for ramjet/scramjet combustion," 43rd AIAA Aerospace Sciences Meeting and Exhibit, Reno, Nevada, January 2005.

[10] Nelson, C. C., "An overview of the NPARC Alliances Wind-US flow solver," AIAA paper, Vol. 27, 2010, pp. 2010.

[11] Steelant, J., Mack, A., Hannemann, K., and Gardner, A., "Comparison of Supersonic Combustion Tests with Shock Tunnels, FLight and CFD," 42nd AIAA/ASME/SAE/ASEE Joint Propulsion Conference and Exhibit, Sacramento, California, July 2006.

[12] Baurle, R. and Eklund, D., "Analysis of dual-mode hydrocarbon scramjet operation at Mach 4-6.5," Journal of Propulsion and Power, Vol. 18, No. 5, 2002, pp. 990-1002.

[13] Drummond, J. P., Danehy, P. M., Gaffney Jr, R. L., Tedder, S. A., Cutler, A. D., and Bivolaru, D., "Supersonic combustion research at NASA," 2007.

[14] Palacios, F., Colonno, M. R., Aranake, A. C., Campos, A., Copeland, S. R., Economon, T. D., Lonkar, A. K., Lukaczyk, T. W., Taylor, T. W., and Alonso, J. J., "Stanford University Unstructured (SU2): An opensource integrated computational environment for multi-physics simulation and design," AIAA Paper, Vol. 287, 2013, pp. 2013.

[15] Palacios, F., Economon, T. D., Aranake, A. C., Copeland, S. R., Lonkar, A. K., Lukaczyk, T. W., Manosalvas, D. E., Naik, K. R., Padrón, A. S., Tracey, B., et al., "Stanford University Unstructured (SU2): Open-source analysis and design technology for turbulent flows," AIAA paper, Vol. 243, 2014, pp. 13-17.

[16] Weller, H. G., Tabor, G., Jasak, H., and Fureby, C., "A tensorial approach to computational continuum mechanics using object-oriented techniques," Computers in physics, Vol. 12, No. 6, 1998, pp. 620-631.

[17] Chapuis, M., Fedina, E., Fureby, C., Hannemann, K., Karl, S., and Schramm, J. M., "A computational study of the HyShot II combustor performance," Proceedings of the Combustion Institute, Vol. 34, No. 2, 2013, pp. 2101-2109. 
[18] Fureby, C., Nordin-Bates, K., Petterson, K., Bresson, A., and Sabelnikov, V., "A computational study of supersonic combustion in strut injector and hypermixer flow fields," Proceedings of the Combustion Institute, Vol. 35, No. 2, 2015, pp. 2127-2135.

[19] Droeske, N., Nizenkov, P., Vellaramkalayil, J. J., von Wolfersdorf, J., Makowka, K., and Sattelmayer, T., "Validation of a Novel OpenFOAM Solver using a Supersonic, Non-reacting Channel Flow," 19th AIAA International Space Planes and Hypersonic Systems and Technologies Conference, 2014, p. 3088.

[20] Makowka, K., Droeske, N., Vellaramkalayil, J. J., von Wolfersdorf, J., and Sattelmayer, T., "Unsteady RANS investigation of a hydrogenfueled staged supersonic combustor with lobed injectors," 19th AIAA International Space Planes and Hypersonic Systems and Technologies Conference, 2014, p. 3215.

[21] Casseau, V., Palharini, R. C., Scanlon, T. J., and Brown, R. E., "A Two-Temperature Open-Source CFD Model for Hypersonic Reacting Flows, Part One: Zero-Dimensional Analysis," Aerospace, Vol. 3, No. 4, 2016, pp. 34.

[22] Casseau, V., Espinoza, D. E., Scanlon, T. J., and Brown, R. E., "A Two-Temperature Open-Source CFD Model for Hypersonic Reacting Flows, Part Two: Multi-Dimensional Analysis," Aerospace, Vol. 3, No. 4, 2016, pp. 45.

[23] Gollan, R. and Jacobs, P., "About the formulation, verification and validation of the hypersonic flow solver Eilmer," International Journal for Numerical Methods in Fluids, Vol. 73, No. 1, 2013, pp. 19-57.

[24] Chan, W., Jacobs, P., and Mee, D., "Suitability of the k- $\omega$ turbulence model for scramjet flowfield simulations," International Journal for Numerical Methods in Fluids, Vol. 70, No. 4, 2012, pp. 493-514.

[25] Sridhar, V., Gai, S., and Kleine, H., "A Numerical Investigation of Supersonic Cavity Flow At Mach 2," 18th Australasian Fluid Mechanics Conference, Launceston, Australia, 2012.

[26] Sridhar, V., Gai, S., and Kleine, H., "Supersonic flow over a rectangular open cavity: effect of length-to-depth ratio," 29th International Symposium on Shock Waves 1, Springer, 2015, pp. 429-434.

[27] Deepak, N., Gai, S., and Neely, A., "Aerothermodynamics of hypersonic shock wave boundary layer interactions," Proc. of 17th Australian Fluid Mechanics Conference, 2010. 
[28] Chan, W. Y., Mee, D. J., Smart, M. K., and Turner, J. C., "Drag Reduction by Boundary-Layer Combustion: Effects of Flow Disturbances from Rectangular-to-Elliptical-Shape-Transition Inlets," Journal of Propulsion and Power, Vol. 31, No. 5, 2015, pp. 1256-1267.

[29] Kang, X., Gollan, R., Jacobs, P., and Veeraragavan, A., "Numerical Simulations of Premixed Combustion in Narrow Channels," 19th Australasian Fluid Mechanics Conference, RMIT University, 2014.

[30] Baurle, R., "Modeling of high speed reacting flows: established practices and future challenges," AIAA paper, Vol. 267, 2004, pp. 2004.

[31] Jacobs, P., Gollan, R., Denman, A., O'Flaherty, B., Potter, D., PetrieRepar, P., and Johnston, I., "Eilmer's theory book: basic models for gas dynamics and thermochemistry," Tech. rep., The University of Queensland, 2012.

[32] Gordon, S. and McBride, B. J., Computer program for calculation of complex chemical equilibrium compositions and applications. I.Analysis, Citeseer, 1996.

[33] Alkandry, H., Boyd, I. D., and Martin, A., "Comparison of models for mixture transport properties for numerical simulations of ablative heat-shields," 51st AIAA Aerospace Sciences Meeting including the New Horizons Forum and Aerospace Exposition, number AIAA, Vol. 303, 2013.

[34] Wilcox, D. C., "Formulation of the k- $\omega$ turbulence model revisited," AIAA journal, Vol. 46, No. 11, 2008, pp. 2823-2838.

[35] Menter, F. R., "Two-equation eddy-viscosity turbulence models for engineering applications," AIAA journal, Vol. 32, No. 8, 1994, pp. 15981605.

[36] Menter, F. and Esch, T., "Elements of industrial heat transfer predictions," 16th Brazilian Congress of Mechanical Engineering (COBEM), Vol. 109, 2001.

[37] Menter, F., Kuntz, M., and Langtry, R., "Ten years of industrial experience with the SST turbulence model," Turbulence, heat and mass transfer, Vol. 4, No. 1, 2003.

[38] Georgiadis, N. J., Yoder, D. A., Vyas, M. A., and Engblom, W. A., "Status of turbulence modeling for hypersonic propulsion flowpaths," Theoretical and Computational Fluid Dynamics, Vol. 28, No. 3, 2014, pp. $295-318$. 
[39] Macrossan, M., "The equilibrium flux method for the calculation of flows with non-equilibrium chemical reactions," Journal of Computational Physics, Vol. 80, No. 1, 1989, pp. 204-231.

[40] Wada, Y. and Liou, M., "A flux splitting scheme with high-resolution and robustness for discontinuities," 32nd Aerospace Sciences Meeting and Exhibit, AIAA-94-0083, Reno, Nevada, 1994.

[41] Liou, M., "Ten years in the making - AUSM-family." AIAA Paper, 2001, pp. 2001-2521.

[42] Coratekin, T., Keuk, J. V., and Ballman, J., "Performance of upwind schemes and turbulence models in hypersonic flows," AIAA journal, Vol. 42, No. 5, 2004, pp. 945-957.

[43] Kurganov, A., Noelle, S., and Petrova, G., "Semidiscrete centralupwind schemes for hyperbolic conservation laws and Hamilton-Jacobi equations," SIAM Journal on Scientific Computing, Vol. 23, No. 3, 2001, pp. $707-740$.

[44] Kurganov, A. and Tadmor, E., "New high-resolution central schemes for nonlinear conservation laws and convection-diffusion equations," Journal of Computational Physics, Vol. 160, No. 1, 2000, pp. 241-282.

[45] Greenshields, C. J., Weller, H. G., Gasparini, L., and Reese, J. M., "Implementation of semi-discrete, non-staggered central schemes in a colocated, polyhedral, finite volume framework, for high-speed viscous flows," International Journal for Numerical Methods in Fluids, Vol. 63, No. 1, 2010, pp. 1-21.

[46] Jacobs, P. and Gollan, R., "Implementation of a compressible-flow simulation code in the D programming language," Applied Mechanics and Materials, Vol. 846, Trans Tech Publ, 2016, pp. 54-60.

[47] Smayda, M., Vogel, P., Schultz, I., Hanson, R., Foelsche, R., Tsai, C., Cresci, D., and Goyne, C., "Hypervelocity Testing of a Dual-mode Scramjet," 50th AIAA Aerospace Sciences Meeting Including the New Horizons Forum and Aerospace Exposition, 2012, p. 481.

[48] Lorrain, P., Brieschenk, S., and Boyce, R., "Experimental investigation of inlet-injection radical-farming scramjet combustion," XXI International Symposium on Air Breathing Engines (ISABE 2013), Vol. 1, Curran Associates, 2013, pp. 1-9.

[49] Tirtey, S., Boyce, R., Brown, M., Capra, B., Creagh, M.and Dedman, A., Dimitrijevic, I., Pudsey, A., Sharp, B., Van Standen, P., et al., "SCRAMSPACE: Radical-Farming Scramjet for access to space," STOEN-AVT-234 Hypersonic Flight Testing, 2014. 
[50] Mogavero, A., Taylor, I., and Brown, R., "Hybrid Propulsion Parametric and Modular Model: a novel engine analysis tool conceived for design optimization," 19th AIAA International Space Planes and Hypersonic Systems and Technologies Conference, Atlanta GA, 2014, pp. $16-20$.

[51] Toro, E. F., Riemann solvers and numerical methods for fluid dynamics: a practical introduction, Springer Science \& Business Media, 2013.

[52] Goldberg, U., Peroomian, O., Chakravarthy, S., and Sekar, B., "Validation of CFD++ code capability for supersonic combustor flowfields," AIAA paper, Vol. 3271, 1997.

[53] Lorrain, P., Flow structure/chemistry coupling in the ignition process in shock-induced-combustion scramjets, Ph.D. thesis, University of Queensland, Australia, 2014.

[54] Jasak, H., Weller, H., and Gosman, A., "High resolution NVD differencing scheme for arbitrarily unstructured meshes," International journal for numerical methods in fluids, Vol. 31, No. 2, 1999, pp. 431-449.

[55] "ARCHIE-WeSt High Performance Computer," https://www . archie-west.ac.uk/, Accessed: 2017-29-01.

[56] Vogel, P., Numerical Simulation of a Hypervelocity Scramjet Engine., Master's thesis, 2012. 